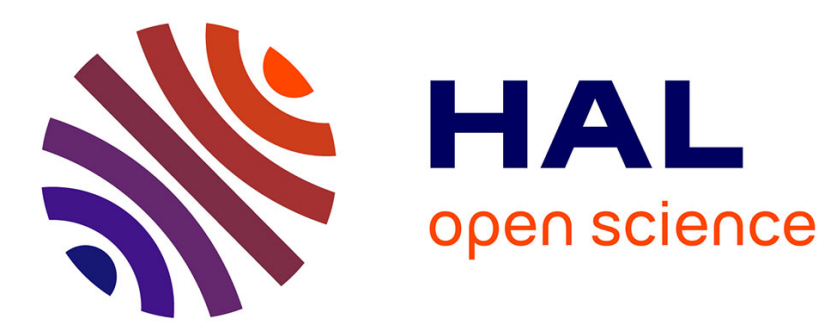

\title{
Modal control based on direct modal parameters estimation
}

\author{
Baptiste Chomette, Adrien Mamou-Mani
}

\section{To cite this version:}

Baptiste Chomette, Adrien Mamou-Mani. Modal control based on direct modal parameters estimation. Journal of Vibration and Control, 2018, 24 (12), pp.2389-2399. 10.1177/1077546317726615 . hal-01821242

\section{HAL Id: hal-01821242 \\ https://hal.sorbonne-universite.fr/hal-01821242}

Submitted on 22 Jun 2018

HAL is a multi-disciplinary open access archive for the deposit and dissemination of scientific research documents, whether they are published or not. The documents may come from teaching and research institutions in France or abroad, or from public or private research centers.
L'archive ouverte pluridisciplinaire HAL, est destinée au dépôt et à la diffusion de documents scientifiques de niveau recherche, publiés ou non, émanant des établissements d'enseignement et de recherche français ou étrangers, des laboratoires publics ou privés. 


\title{
Modal control based on direct modal parameters estimation
}

\author{
Baptiste Chomette ${ }^{1,2}$ and Adrien Mamou-Mani ${ }^{1,3}$
}

June 21, 2018

\author{
1. Sorbonne Universités, UPMC Univ Paris 06 \\ 2. CNRS, UMR 7190, Institut Jean le Rond d'Alembert, F-75005, Paris, France \\ 3. IRCAM, CNRS, UMR 9912, STMS, F-75004, Paris, France \\ Corresponding author: baptiste.chomette@upmc.fr
}

\begin{abstract}
Modal active control is based on a state model that requires the identification of modal parameters. This identification can typically be done through a rational fraction polynomial algorithm applied in the frequency domain. This method generates numerical problems when estimating high-order models, particularly when moving from the basis of orthogonal polynomials for the modal basis. This algorithm must therefore be applied independently on multiple frequency ranges with a low order for each range. In this case, the controller design cannot be automated and requires a lot of human intervention especially to build the state space model. To address this issue, this paper presents the application of the direct modal parameters estimation algorithm for active modal control design. The identification algorithm is presented in a simplified version with only positive frequencies. Unlike other classical identification methods in the frequency domain, the direct modal parameters estimation algorithm provides a solution with a great numerical stability and allows estimating models with a higher order. Using this method, the design of the controller can be largely automated and requires a minimal of human intervention. After a theoretical presentation, the proposed method is experimentally validated by controlling the vibration modes of a suspended plate.
\end{abstract}

\section{Introduction}

In current industrial structures, severe stress conditions like high-level vibrations cause robustness problems and affect the lifetime of structures. One way to reduce vibrations is to apply active control to increase the natural damping of systems and consequently its lifetime. A review of different control techniques such as collocated control, active and passive strategies, state space approach and modal control can be found in [1]. There are many active control applications on different industrial structures like aircraft [2], manipulator [3], multi-axis systems [4] or on shell structures [5]. The application of active modal control is less common cause of the complexity of design and of its lack of robustness. Nevertheless, there are a few applications in the industrial domain. For example, in aerospace domain to reduce damage induced by vibrations on electronic systems [6, 7], in the field of sound transmission [8] or in musical domain where active modal control has become a way to modify the sound of instruments $[9,10]$. Recently, a new approach to the synthesis of active modal control is introduced in [11]. This approach permits to easily evaluate spillover effects on non-modeling modes.

Modal active control is based on a state model that requires the identification of modal parameters, frequency, damping, actuators and sensors properties (modal amplitudes). There are a lot of identification algorithms in the frequency domain which can be used to achieve it. The traditional rational fraction polynomial algorithm (RFP) [12] based on the use of orthogonal polynomials [13, 14] is a first solution. A control procedure based on the RFP algorithm is detailed in [15]. This procedure is based on the identification of the product of actuator and sensor properties. Indeed, it's important to note that just this product has to be identified to establish the controller. There is no need to identify independently the actuator and sensor matrices. For example, in the case of piezoelectric sensors and actuators, the measure of the electromechanical properties of the transducers is very difficult without a dedicated system $[16,17]$. In spite of its application, the RFP algorithm is not suitable in the case of high modal density 
or high model order. In this case, the identification of modal parameters is in general not optimal due to the ill-conditioned problem to estimate the power polynomial matrices from the orthogonal polynomial matrices. One approach that is used to avoid this problem consists in cutting the spectrum in several frequency ranges that need only a few modes in the identification procedure. But this solution does no permit to automate the control procedure. An alternative approach consists in building an identification algorithm based on well conditioned matrices for higher order model. Recently, the dual fitting method [18] or the Direct Modal Parameters Estimation (DMPE) [19] propose a stable numerical solution to estimate high order models. The DMPE algorithm is introduced using complex orthogonal polynomials with both negative and positive frequencies for the generation of orthogonal polynomials as explained in [13]. The principle of the method is based on companion matrices introduced in ([20]). Moreover, in control procedures, the interpretation of identification results has to be automated. One way to select physical modal parameters is to use stabilization charts as explained in [21] and [22].

In this paper, we propose a new control procedure based on the DMPE algorithm and using automated stabilization charts interpretation to design the state model of the controller. The formulation of the DMPE algorithm is simplified using real polynomials with only positive frequencies. The identification results will be then included in a control procedure. This paper is organized as follows. The identification and the control design are theoretically presented in the first section. The efficiency of the proposed method is then experimentally validated and compared to the classical procedure based on the RFP algorithm in the second section.

\section{Identification and control design}

The aim of the identification step is to determine the state model of the structure which can be described by

$$
\left\{\begin{array}{l}
\dot{\mathbf{x}}(t)=\mathbf{A x}(t)+\mathbf{B} u(t)+\mathbf{G} w(t) \\
\mathbf{y}(t)=\mathbf{C x}(t)
\end{array},\right.
$$

where $\mathbf{A}$ is the dynamical system matrix, $\mathbf{B}$ and $\mathbf{C}$ are the actuator and sensor matrices respectively and $\mathbf{G}$ is the disturbance matrix. $\mathbf{x}$ and $\mathbf{y}$ are the state and the output vectors respectively. $u$ is the control and $w$ the disturbance. In the case of one actuator and one disturbance point, $u$ and $w$ are scalar quantities. The state vector can be written for $n$ modes

$$
\mathbf{x}=\left[\begin{array}{c}
\mathbf{q} \\
\dot{\mathbf{q}}
\end{array}\right],
$$

where $\mathbf{q}$ is the modal displacement vector. The state matrices can be written for $\mathrm{n}$ modes

$$
\begin{array}{r}
\mathbf{A}=\left[\begin{array}{cc}
\mathbf{0}_{n \times n} & \mathbf{I d}_{n \times n} \\
-\operatorname{diag}\left(\omega_{k}^{2}\right)_{n \times n} & -\operatorname{diag}\left(2 \xi_{k} \omega_{k}\right)_{n \times n}
\end{array}\right]_{2 n \times 2 n}, \\
\mathbf{B}=\left[\begin{array}{c}
\mathbf{0}_{n \times 1} \\
\Pi^{a}{ }_{n \times 1}
\end{array}\right]_{2 n \times 1}, \mathbf{C}=\left[\begin{array}{ll}
\Pi^{s}{ }_{1 \times n} & \mathbf{0}_{1 \times n}
\end{array}\right]_{1 \times 2 n}, \mathbf{G}=\left[\begin{array}{c}
\mathbf{0}_{n \times 1} \\
\Pi^{w}{ }_{n \times 1}
\end{array}\right]_{2 n \times 1},
\end{array}
$$

where $\omega_{k}$ and $\xi_{k}$ are the modal frequency and damping respectively. $\Pi^{a}, \Pi^{w}$ and $\Pi^{s}$ are the actuator, sensor and disturbance vectors respectively.

This model is then used to design the observer and the controller. The crucial point of the identification is the numerical stability of the procedure, that's why we propose to use the DMPE algorithm to identify the state model of the structure.

\subsection{Simplified DMPE algorithm}

The measured transfer function between one sensor output $y$ and one actuator input $u$ can be expressed in a rational form using orthogonal polynomials and for $n$ modes

$$
H\left(s_{i}\right)=\frac{y}{u}=\frac{\sum_{k=0}^{2 n-1} \beta_{k} \phi_{i, k}\left(s_{i}\right)}{\sum_{k=0}^{2 n} \alpha_{k} \theta_{i, k}\left(s_{i}\right)},
$$


where $s_{i}=j \omega_{i}$ is the Laplace variable evaluated at frequency $\omega_{i} . \quad \phi_{i, k}$ and $\theta_{i, k}$ are the numerator and denominator orthogonal polynomials matrix associated to the coefficients $\beta_{k}$ and $\alpha_{k}$ respectively for frequency $i$ and order $k$. These coefficients are supposed to be estimated from a first step. More details are given in $[13,14,12]$. We remind that the zeros of the denominators are the poles of the system associated to the modal parameters, frequency and damping. The generation of complex Forsythe polynomials for negative and positive frequencies using a recurrence relation is detailed in [13]. The simplification of this method using real polynomials and just positive frequencies is explained in [12]. In the case of Forsythe polynomials, the real orthogonal polynomials $S_{i, k}$ of the numerator can be calculated for a complex Frequency Response Function (FRF) of length $L$ using the recurrence relation

$$
S_{i, k}=\omega_{i} R_{i, k-1}-V_{k-1} R_{i, k-2},
$$

where

$$
V_{k-1}=2 \sum_{i=1}^{L} \omega_{i} R_{i, k-1} R_{i, k-2} q_{i}
$$

The normalized real Forsythe polynomial is obtained from

$$
R_{i, k}=\frac{S_{i, k}}{D_{k}}
$$

with

$$
D_{k}=\left(2 \sum_{i=1}^{L} S_{i, k}^{2} q_{i}\right)^{1 / 2}
$$

The complex polynomials $P_{i, k}$ are obtained from the real Forsythe polynomials using

$$
P_{i, k}=(j)^{k} R_{i, k} .
$$

Equation (5) can be written using (7)

$$
\omega_{i} R_{i, k-1}=D_{k} R_{i, k}+V_{k-1} R_{i, k-2} .
$$

Multiply the above through by $j^{k}$ and with equation (9), the recurrence relation can be written

$$
\left(j \omega_{i}\right) P_{i, k-1}=D_{k} P_{i, k}-V_{k-1} P_{i, k-2}
$$

and finally

$$
P_{i, k}=s \frac{P_{i, k-1}}{D_{k}}+V_{k-1} \frac{P_{i, k-2}}{D_{k}} .
$$

This relation is available for the denominator using $P_{i, k}=\theta_{i, k}$. The $2 n$ poles of the system can be found by seeking the roots of the equation

$$
\sum_{k=0}^{2 n} \alpha_{k} \theta_{i, k}\left(s_{i}\right)=0 .
$$

Using the polynomial fraction-matrix model and the fact that $\alpha_{2 n}=1$, the roots of the denominator of the transfer function (4) can be calculated with

$$
\sum_{k=0}^{2 n} \alpha_{k} \theta_{i, k}\left(s_{i}\right)=0 \Leftrightarrow \theta_{i, 2 n}=-\sum_{k=0}^{2 n-1} \alpha_{k} \theta_{i, k}\left(s_{i}\right) .
$$

Equation (14) can be written in an equivalent matrix form

$$
\begin{array}{r}
{\left[\begin{array}{llll}
\theta_{i, 2 n} & \theta_{i, 2 n-1} & \cdots & \theta_{i, 1}
\end{array}\right]_{1 \times 2 n}=} \\
{\left[\begin{array}{llll}
\theta_{i, 2 n-1} & \theta_{i, 2 n-2} & \cdots & \theta_{i, 0}
\end{array}\right]_{1 \times 2 n} \mathbf{D}_{d e n}}
\end{array}
$$

with

$$
\mathbf{D}_{d e n}=\left[\begin{array}{cccccc}
-\alpha_{2 n-1} & 1 & 0 & . & . & 0 \\
-\alpha_{2 n-2} & 0 & 1 & . & . & 0 \\
\cdot & . & . & . & . & . \\
\cdot & . & . & . & . & . \\
-\alpha_{1} & 0 & . & . & 1 & 0 \\
-\alpha_{0} & 0 & . & . & 0 & 0
\end{array}\right]_{2 n \times 2 n}
$$


Using recurrence relation (12) applied for the denominator with $P_{i, k}=\theta_{i, k}$

$$
\begin{aligned}
& {\left[\begin{array}{llll}
\theta_{i, 2 n} & \theta_{i, 2 n-1} & \cdots & \theta_{i, 1}
\end{array}\right]_{1 \times 2 n}=} \\
& {\left[\begin{array}{llll}
\theta_{i, 2 n-1} & \theta_{i, 2 n-2} & \cdots & \theta_{i, 0}
\end{array}\right]_{1 \times 2 n}\left(s \mathbf{Z}_{d e n}+\mathbf{P}_{d e n}\right)}
\end{aligned}
$$

with

$$
\begin{aligned}
& \mathbf{Z}_{d e n}=\left[\begin{array}{ccccc}
D_{2 n}^{-1} & 0 & \cdot & \cdot & 0 \\
0 & D_{2 n-1}^{-1} & 0 & \cdot & 0 \\
\cdot & \cdot & \cdot & \cdot & \cdot \\
\cdot & \cdot & \cdot & \cdot & \cdot \\
\cdot & \cdot & \cdot & \cdot & D_{1}^{-1}
\end{array}\right]_{2 n \times 2 n}, \\
& \mathbf{P}_{d e n}=\left[\begin{array}{ccccc}
0 & \cdot & \cdot & \cdot & 0 \\
V_{2 n-1} D_{2 n}^{-1} & 0 & \cdot & \cdot & 0 \\
\cdot & \cdot & \cdot & \cdot & \cdot \\
\cdot & \cdot & \cdot & \cdot & \cdot \\
0 & \cdot & \cdot & V_{2} D_{2}^{-1} & 0
\end{array}\right]_{2 n \times 2 n} .
\end{aligned}
$$

Equation (17) can be written in the form of an eigenvalue problem

$$
\mathbf{D}_{d e n}=s \mathbf{Z}_{d e n}+\mathbf{P}_{d e n} \Leftrightarrow\left(\mathbf{D}_{d e n}-\mathbf{P}_{d e n}\right) \mathbf{Z}_{d e n}^{-1}-s \mathbf{I d}=\mathbf{0} .
$$

In practice, the estimation is achieved using scaled frequency to avoid numerical problems. The simplest scaling procedure is to scale all frequency to the unit interval by dividing all frequency by the highest value $\omega_{\max }$. The eigenvalues $\lambda_{k}$ and its conjugate $\lambda_{k}{ }^{*}$ of the matrix $\mathbf{G}_{d e n}=\left(\mathbf{D}_{d e n}-\mathbf{P}_{d e n}\right) \mathbf{Z}_{d e n}^{-1}$ can be written using the scaled frequency $\bar{w}_{k}$ and modal damping $\bar{\xi}_{k}$

$$
\lambda_{k}, \lambda_{k}^{*}=-\xi_{k} \bar{\omega}_{k} \pm j \bar{\omega}_{k} \sqrt{1-\bar{\xi}_{k}^{2}} .
$$

The modal frequency $\omega_{k}$ and modal damping $\xi_{k}$ can be finally obtained using

$$
\omega_{k}=\left|\lambda_{k}\right| \omega_{\max }, \xi_{k}=-\frac{\operatorname{Re}\left(\lambda_{k}\right) \omega_{\max }}{\omega_{k}} .
$$

\subsection{Selection of the stable poles and modal amplitude coefficients estimation}

The stable poles are then automatically extracted using a stabilization chart. We remind that a stabilization chart is based on several runs of the pole identification process by using models of increasing order. Physical poles always appears at a nearly frequency whereas mathematical poles tend to scatter around the frequency range. The typical stabilization criteria are chosen as equal to $1 \%$ for the frequency and $5 \%$ for the damping between two runs at order $n$ and $n+1$. The identified transfer function can then be written in terms of stable modal parameters corresponding to physical poles

$$
H_{D M P E}(s)=\sum_{k=0}^{n}\left(\frac{R_{k}}{s^{2}+2 s \xi_{k} \omega_{k}+\omega_{k}^{2}}\right),
$$

where $R_{k}$ denotes the complex modal amplitudes which can be calculated using the linear square problem evaluated at each frequency $\omega_{i}$. The optimal order is automatically determined using the error criterion $\varepsilon$ between the measured $\left(f r f_{m}\right)$ and the estimated $\left(f r f_{e}\right)$ FRF obtained from the stable poles

$$
\varepsilon=\frac{\sum_{i=1}^{L}\left(\left\|f r f_{e}\right\|-\left\|f r f_{m}\right\|\right)^{2}}{\sum_{i=1}^{L}\left(\left\|f r f_{m}\right\|^{2}\right)} .
$$




\subsection{From modal parameters to state space model}

The transfer function between the control $u$ applied on the actuator and the output $y$ measured on one sensor can be written in a matrix format and without disturbance

$$
H(s)=\frac{y}{u}=\mathbf{C}(s \mathbf{I} \mathbf{d}-\mathbf{A})^{-1} \mathbf{B}
$$

where $s$ denotes the Laplace variable. This transfer function can also be written using the modal parameters summing up each vibration mode

$$
H(s)=\sum_{k=0}^{n}\left(\frac{\Pi_{k}^{a} \Pi_{k}^{s}}{s^{2}+2 s \xi_{k} \omega_{k}+\omega_{k}^{2}}\right),
$$

where the numerator $\Pi_{k}^{a} \Pi_{k}^{s}$ is a real quantity. The modal frequency and damping of equation (25) are directly obtained using the denominator of equation (22) and can be used to define the dynamical system matrix A.

The product value $\Pi_{k}^{a} \Pi_{k}^{s}$ can be identify using the complex numerator $R_{k}$ of equation (22) which can be written using its modulus and phase

$$
R_{k}=\left|R_{k}\right|\left(\cos \left(\varphi_{k}\right)+j \sin \left(\varphi_{k}\right)\right)
$$

where $\varphi_{k}$ denotes the phase angle. In the case of a lightly damped system with a diagonal damping matrix, the phase angle can be supposed equal to $\pm \pi$ and the complex numerator becomes a real quantity

$$
R_{k}=\left|R_{k}\right| \cos ( \pm \pi)
$$

The sign of the numerator is determined by the sign of the cosinus and consequently by the sign of the real part of the numerator $\Re\left(R_{k}\right)$. The numerator can then be written

$$
R_{k}=\left|R_{k}\right| \operatorname{sign}\left(\Re\left(R_{k}\right)\right) \text {. }
$$

Finally, the product of the actuator and sensor modal coordinates can be identified using

$$
\Pi_{k}^{a} \Pi_{k}^{s}=\left|R_{k}\right| \operatorname{sign}\left(\Re\left(R_{k}\right)\right) .
$$

The actuator and sensors matrices are finally obtained with

$$
\begin{array}{r}
\mathbf{B}=\left[\begin{array}{c}
\mathbf{0}_{n \times 1} \\
\left|R_{k}\right| \operatorname{sign}\left(\Re\left(R_{k}\right)\right)_{n \times 1}
\end{array}\right]_{2 n \times 1}, \\
\mathbf{C}=\left[\begin{array}{ll}
\mathbf{1}_{1 \times n} & \mathbf{0}_{1 \times n}
\end{array}\right]_{1 \times 2 n} .
\end{array}
$$

If the assumption about damping is not completely verified, the imaginary part of the complex numerator is reflected in a phase shift on the real modal displacement. This phase shift may have an impact on the control performances.

\subsection{Controller and observer design}

The modal control is classically design using a pole placement algorithm [23] for both the control gain $\mathbf{K}$ and the observer gain $\mathbf{L}$ used in the Luenberger observer [24]. The control $u$ is obtained from the estimated state vector $\hat{\mathbf{x}}$ with

$$
u(t)=-\mathbf{K} \hat{\mathbf{x}}(t)
$$

The dynamic of the controller without disturbance and including the observer is defined by

$$
\left\{\begin{array}{l}
\dot{\hat{\mathbf{x}}}(t)=\mathbf{A}_{m} \hat{\mathbf{x}}(t)+\mathbf{B}_{m} u(t)+\mathbf{L}(\mathbf{y}(t)-\hat{\mathbf{y}}(t)) \\
\hat{\mathbf{y}}(t)=\mathbf{C}_{m} \hat{\mathbf{x}}(t)
\end{array}\right.
$$

where $\hat{\mathbf{y}}$ is the estimated output vector. The subscription $m$ denotes the matrix used in the observer and build up using the DMPE identification. 


\section{Application}

The proposed method is applied to a suspended $250 \times 380 \times 3.5 \mathrm{~mm}$ rectangular plate composed of Duralumin and presented in Figure 1(a). The structure is instrumented with one exciter HIAX19C01-8 and one collocated piezoelectric PZT sensor located behind the plate. This structure was chosen for its rich non harmonic sound properties close to a bell plate sound. The acquisition and control loop are carried out using a COALA system, presented in Figure 1(b), including a signal pre-amplifier, a $20 \mathrm{~W}$ power amplifier and a real time control system based on the real time frame work Xenomai on a BeagleBone Black [25].

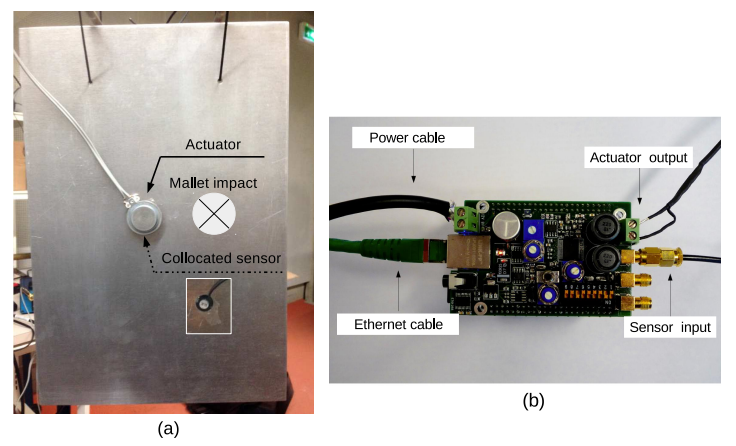

Figure 1: Studied plate (a) and control system COALA (b).

\subsection{RFP versus DMPE identification}

In this section, we compare the identification procedures using the RFP and the DMPE algorithm. The plate is excited with a swept sine applied on the actuator. The output is measured on the piezoelectric sensor. The complex FRF between the actuator and the sensor is used to applied the two identification algorithms.

\subsubsection{RFP identification}

A first identification is realized using the RFP algorithm on the single frequency range 100-1735 Hz. The stabilization chart presented in Figure 2 is achieved for an order varying from $n=10$ to $n=50$. To make it clearer, only the stable poles in frequency and damping marked by ' $\bullet$ ' are represented on the chart. The optimal order is 24 and the identified modal parameters (frequency $f_{k}$, modal damping $\xi_{k}$ and amplitudes $R_{k}$ ) are detailed in Table 1. Only three modes are identified but the algorithm do not keep the numerical stability when the order increase more than 24 .

Table 1: Modal parameters identified using the RFP algorithm on the frequency range $100-1735 \mathrm{~Hz}$ for $\mathrm{n}=24$.

\begin{tabular}{cccc}
\hline Mode & $f_{k}[\mathrm{~Hz}]$ & $\xi_{k}[\%]$ & $R_{k}$ \\
\hline 1 & 148.7 & 0.09 & $-2.1 \times 10^{4}-6.6 \times 10^{3} \mathrm{j}$ \\
4 & 690.3 & 0.04 & $-1.2 \times 10^{5}+6.0 \times 10^{4} \mathrm{j}$ \\
5 & 839.4 & 0.06 & $-1.9 \times 10^{5}+1.4 \times 10^{5} \mathrm{j}$ \\
\hline
\end{tabular}

As explained in the introduction, a solution to identify more modes consists in cutting the spectrum in several frequency ranges that need only a few modes in the identification procedure. Results corresponding to this second identification are detailed in Table 2 . Six different frequency ranges are needed to identify modes 1, 2, 3, 4, 5, 6, 7 and 8: 100-350 $\mathrm{Hz}$ for mode 1, 350-500 $\mathrm{Hz}$ for mode 2, 300-700 $\mathrm{Hz}$ for mode 3, 600-1000 Hz for modes 4 and 5, 1200-1745 Hz for modes 6 and 8 and finally 1370-1490 $\mathrm{Hz}$ for mode 7 . In this case, the identification procedure can not be automated and requires a lot of adjustments.

\subsubsection{DMPE identification}

A third identification is realized using the DMPE algorithm on the single frequency range 100-1735 Hz. The stabilization chart is presented in Figure 2 (' + ' denotes the stable poles). The optimal order is 47 
Table 2: Modal parameters identified using the RFP algorithm on several frequency ranges.

\begin{tabular}{cccccc}
\hline Mode & $f_{k}[\mathrm{~Hz}]$ & $\xi_{k}[\%]$ & $R_{k}$ & Frequency range & Optimal order \\
\hline 1 & 148.7 & 0.15 & $-2.7 \times 10^{4}-3.9 \times 10^{3} \mathrm{j}$ & $100-350 \mathrm{~Hz}$ & 12 \\
2 & 360.8 & 0.08 & $-5.8 \times 10^{4}-5.8 \times 10^{3} \mathrm{j}$ & $350-500 \mathrm{~Hz}$ & 12 \\
3 & 477.4 & 0.15 & $-1.8 \times 10^{3}-2.6 \times 10^{3} \mathrm{j}$ & $300-700 \mathrm{~Hz}$ & 18 \\
4 & 690.3 & 0.04 & $-1.2 \times 10^{5}+6.3 \times 10^{4} \mathrm{j}$ & $600-1000 \mathrm{~Hz}$ & 11 \\
5 & 839.4 & 0.07 & $-2.0 \times 10^{5}+1.5 \times 10^{5} \mathrm{j}$ & $600-1000 \mathrm{~Hz}$ & 11 \\
6 & 1327.2 & 0.05 & $-1.3 \times 10^{4}+3.5 \times 10^{4} \mathrm{j}$ & $1200-1745 \mathrm{~Hz}$ & 12 \\
7 & 1382.0 & 0.03 & $-8.6 \times 10^{3}+1.4 \times 10^{4} \mathrm{j}$ & $1370-1490 \mathrm{~Hz}$ & 06 \\
8 & 1467.9 & 0.05 & $-1.3 \times 10^{5}+4.1 \times 10^{5} \mathrm{j}$ & $1200-1745 \mathrm{~Hz}$ & 12 \\
\hline
\end{tabular}

and 8 modes are identified. The modal parameters are detailed in Table 3 . We see that the numerical stability of the DMPE algorithm permits to easily increase the order of the identification on the single frequency range to include more modes than using the RFP algorithm. These results are very similar to those from the RFP algorithm applied on several frequency ranges. In this case, the identification procedure can be automated and requires a minimum number of adjustable parameters: the frequency and the order range to build the stabilization chart.

Table 3: Modal parameters identified using the DMPE algorithm on the frequency range 100-1735 $\mathrm{Hz}$ for $\mathrm{n}=47$.

\begin{tabular}{cccc}
\hline Mode & $f_{k}[\mathrm{~Hz}]$ & $\xi_{k}[\%]$ & $R_{k}$ \\
\hline 1 & 148.7 & 0.13 & $-2.5 \times 10^{4}-4.4 \times 10^{3} \mathrm{j}$ \\
2 & 360.8 & 0.07 & $-5.7 \times 10^{4}-5.2 \times 10^{3} \mathrm{j}$ \\
3 & 477.7 & 0.22 & $-2.0 \times 10^{3}-2.9 \times 10^{3} \mathrm{j}$ \\
4 & 690.3 & 0.04 & $-1.2 \times 10^{5}+6.4 \times 10^{4} \mathrm{j}$ \\
5 & 839.4 & 0.07 & $-2.0 \times 10^{5}+1.5 \times 10^{5} \mathrm{j}$ \\
6 & 1327.2 & 0.05 & $-1.5 \times 10^{4}+3.3 \times 10^{4} \mathrm{j}$ \\
7 & 1382.0 & 0.03 & $-8.6 \times 10^{3}+1.7 \times 10^{4} \mathrm{j}$ \\
8 & 1467.0 & 0.05 & $-1.2 \times 10^{5}+4.1 \times 10^{5} \mathrm{j}$ \\
\hline
\end{tabular}
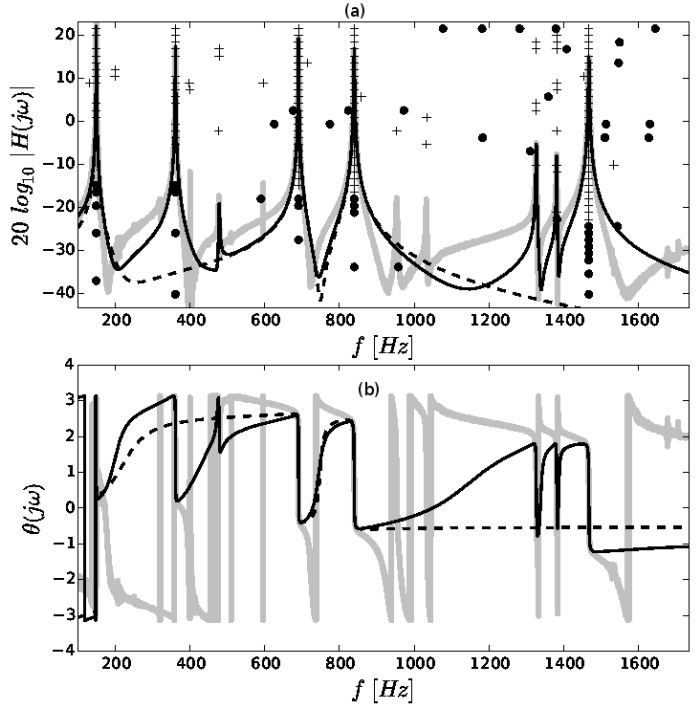

Figure 2: Transfer function (a) and phase angle (b) of the system ( - ); stabilization chart obtained from the RFP $(\bullet)$ and from the DMPE algorithm (+); synthesized FRF obtained from the RFP (- - -) and from the DMPE (—) modal parameters. 


\subsection{Controller and observer design}

The controller and the observer focus on the vibration modes with a maximum amplitude located in the center of the plate: modes 1, 2, 4, 5 and 8 in Table 3 . The other modes correspond to corner modes and are not taken into account in this example. The numerical FRF obtained from the reduced state space model with real modal amplitudes based on equation (30) is presented in Figure 3.
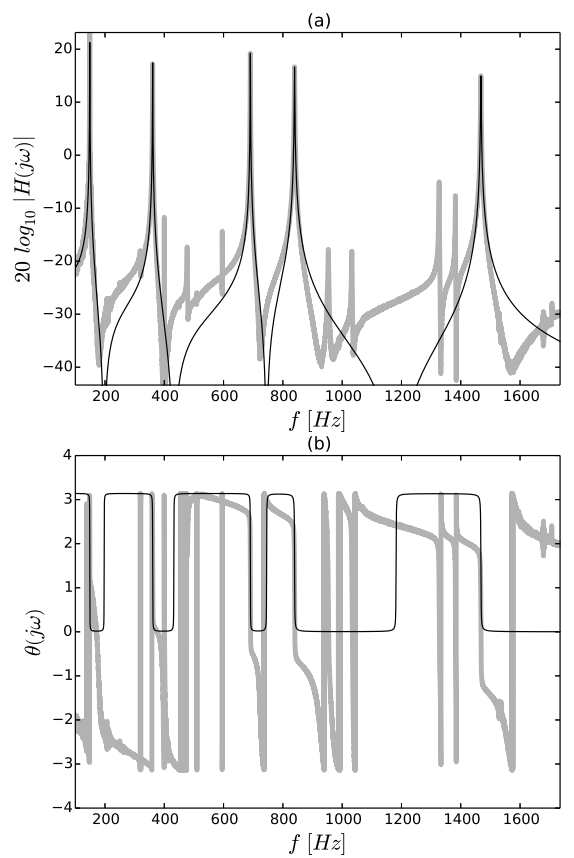

Figure 3: Transfer function (a) and phase angle (b) of the system ( - ) and numerical FRF (a) and phase angle (b) obtained from the reduced state space model with real modal amplitudes

Poles 1, 2, 4, 5 and 8 whose frequencies are equal to $149,361,690,839$ and $1468 \mathrm{~Hz}$ respectively are damped with a coefficient 10 applied on the real part. The control gain $\mathbf{K}_{1 \times 2 n}=\left[\mathbf{0}_{1 \times n} \mathbf{K}_{2}\right]$ and the observer gain $\mathbf{L}_{2 n \times 1}=\left[\mathbf{L}_{1} \mathbf{L}_{2}\right]^{t}$ are conventionally calculated using a pole placement algorithm. The actuator matrix $\mathbf{B}_{m}$, the control and observer gains are detailed in Table 4. The poles of the uncontrolled system corresponding to the eigenvalues of $\mathbf{A}_{m}$ and the poles of the controlled system associated to the eigenvalues of $\left(\mathbf{A}_{m}-\mathbf{B}_{m} \mathbf{K}\right)$ are shown on a pole chart in Figure 4.

Table 4: Actuator matrix, control and observer gains.

\begin{tabular}{ccccc}
\hline Mode & $\mathbf{B}_{m}$ & $\mathbf{K}_{2}$ & $\mathbf{L}_{1}$ & $\mathbf{L}_{2}$ \\
\hline 1 & $-2.5 \times 10^{4}$ & $3.4 \times 10^{-3}$ & $1.4 \times 10^{2}$ & $-4.9 \times 10^{3}$ \\
2 & $-5.7 \times 10^{4}$ & $-2.3 \times 10^{-3}$ & $2.0 \times 10^{2}$ & $3.1 \times 10^{3}$ \\
4 & $-1.4 \times 10^{5}$ & $1.7 \times 10^{-2}$ & $2.1 \times 10^{2}$ & $-1.0 \times 10^{5}$ \\
5 & $-2.5 \times 10^{5}$ & $-3.4 \times 10^{-2}$ & $4.0 \times 10^{2}$ & $3.5 \times 10^{5}$ \\
8 & $-4.3 \times 10^{5}$ & $-3.6 \times 10^{-2}$ & $4.9 \times 10^{2}$ & $6.5 \times 10^{5}$ \\
\hline
\end{tabular}

\subsection{Application with a collocated swept sine excitation}

In this first step, a swept sine is applied to the actuator. The uncontrolled and controlled FRF of the system are presented in Figure 5. A zoom on modes 4 and 5 for the numerical and for the experimental FRF is presented in Figure 6 and 7 respectively. Frequency $f_{k_{c}}$ and damping $\xi_{k_{c}}$ of the controlled system are obtained using the DMPE algorithm applied on the controlled FRF. Values are detailed in Table 5. The frequency shift induced by the controller is very low and remains under $1.1 \%$ for the controlled modes. The increase of damping induced by the controller should be equal to 10 . The controller performances 
are closed to this value for modes $1,2,4$ and 5 but significantly worse for mode 8 with a coefficient of increase only equal to 4.6. This can be explained by the value of its modal amplitudes. In the case of mode 8 , the imaginary part of the modal amplitude is higher than the real part and the phase shift of the modal displacement is not properly taking into account. This error must have a significant impact on the control performances.

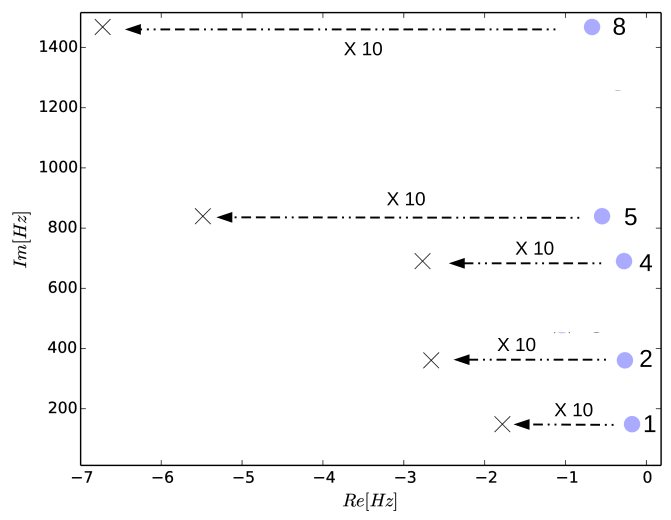

Figure 4: Poles chart of the uncontrolled system $(\bullet)$ and of the controller $(\times)$.
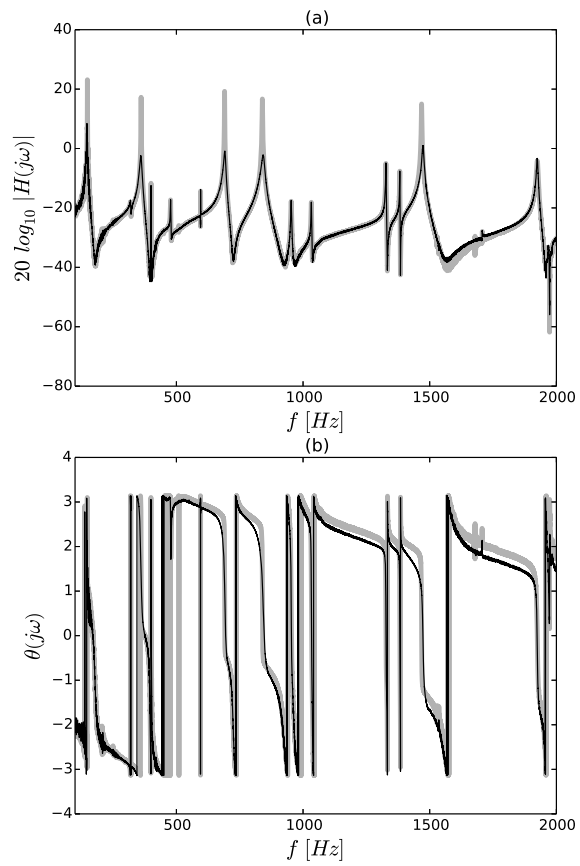

Figure 5: Experimental uncontrolled (—) and controlled (—) FRF (a) and phase angle (b) using the DMPE modal model and a swept sine excitation.

\subsection{Application with a non-collocated percussive excitation}

In a second experimental case, the controller is tested with a percussive excitation, applied with a mallet reproducing the gesture of a percussionist as indicated in Figure 1. The aim of this experiment is to validate the control system in real life context, for any unknown and non repeatable excitation, as in a musical context. The Experimental uncontrolled and controlled sensor signal $y(t)$ using the DMPE modal model and a mallet excitation are shown in Figure 8. The effect of the controller is clearly visible on the 

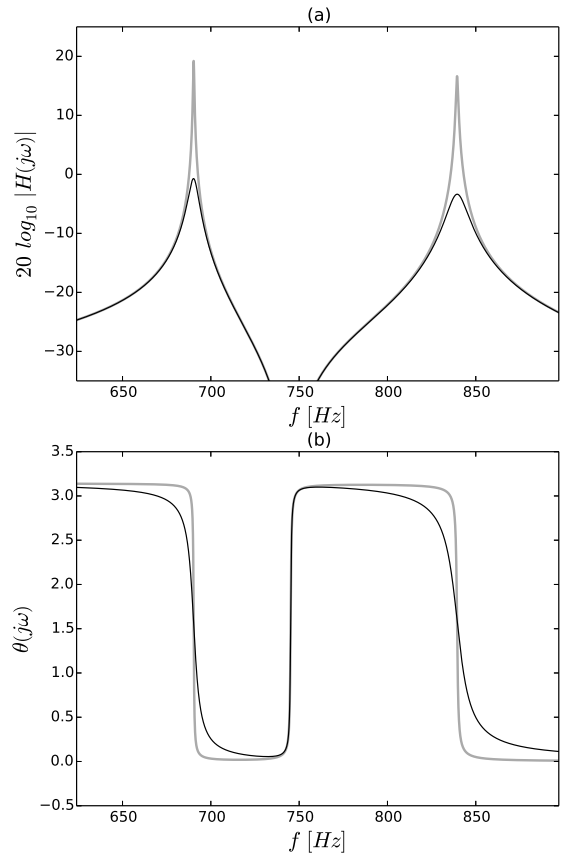

Figure 6: Numerical uncontrolled ) and controlled

FRF (a) and phase angle (b) using the DMPE modal model and a swept sine excitation, zoom on modes 4 and 5 .
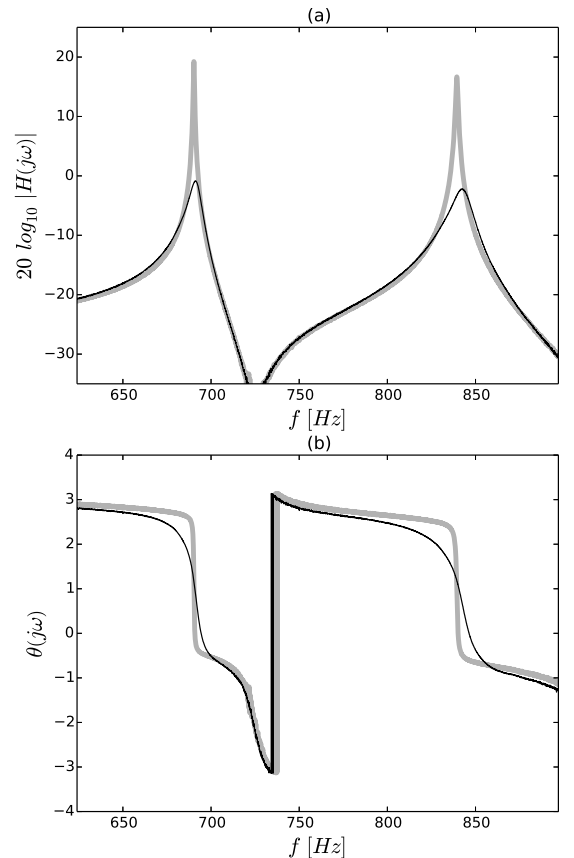

Figure 7: Experimental uncontrolled ) and controlled FRF (a) and phase angle (b) using the DMPE modal model and a swept sine excitation, zoom on modes 4 and 5 .

time signal and shows the amount of damping induce by the controller. We can note that the amplitude of the second percussive excitation is slightly larger than the first one, which explains that the amplitude after control is also slightly larger. The spectrum of the sensor signal in $\mathrm{dB}$ for the uncontrolled and controlled system is shown in Figure 9. These results show a large decrease of the amplitude of controlled modes $1,2,4,5$ and 8 . A subjective analysis of the authors' perceived sounds showed that the metal plate sounds like a wooden plate. 
Table 5: Frequency and damping of the controlled system and coefficient of the damping increase.

\begin{tabular}{cccc}
\hline Mode & $f_{k_{c}}[\mathrm{~Hz}]$ & $\xi_{k_{c}}[\%]$ & $\frac{\xi_{k_{c}}}{\xi_{k}}$ \\
\hline 1 & 147.2 & 1.11 & 8.5 \\
2 & 360.1 & 0.85 & 12.1 \\
4 & 691.5 & 0.47 & 11.7 \\
5 & 842.8 & 0.66 & 9.4 \\
8 & 1473.5 & 0.23 & 4.6 \\
\hline
\end{tabular}

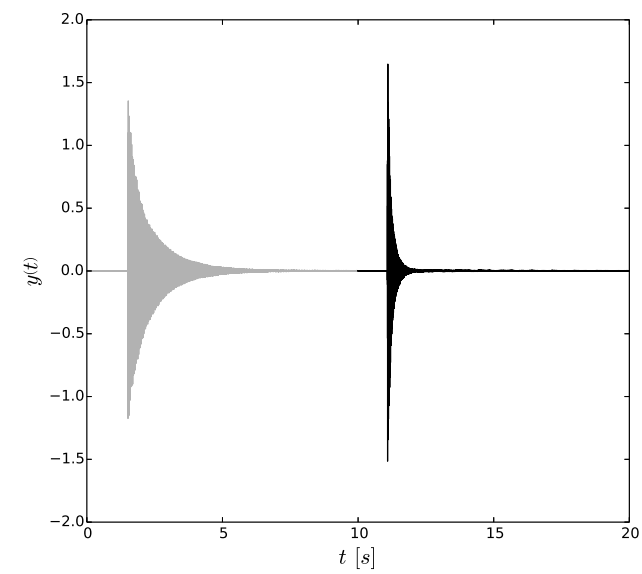

Figure 8: Experimental uncontrolled ( $(-)$ and controlled sensor signal ( $(-)$ using the DMPE modal model and a mallet excitation.

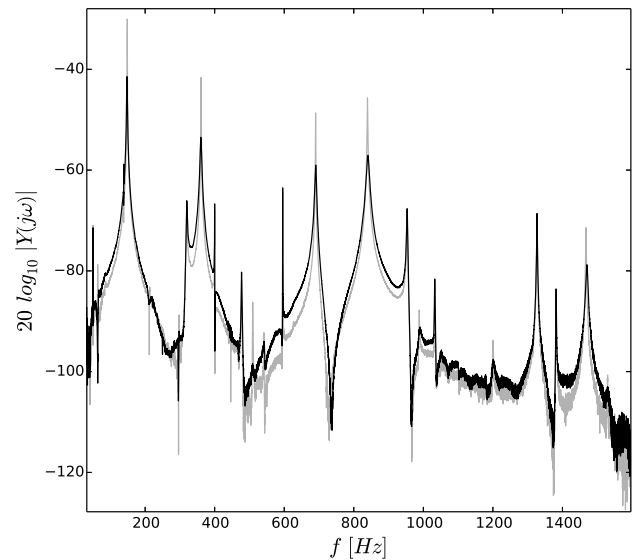

Figure 9: Experimental uncontrolled ( ) and controlled spectrum in $\mathrm{dB}$ of the sensor signal using the DMPE modal model and a mallet excitation.

\section{Summary}

This paper deals with the use of the DMPE algorithm to design active modal control. The presented procedure is particularly efficient and requires a minimum of adjustments. Moreover, the DMPE algorithm is presented here in a simplified version with only positive frequencies and real polynomials. The procedure is experimentally validated on a suspended rectangular plate firstly with a collocated swept sine excitation and secondly with a non-collocated percussive excitation. For the identification procedure, in the case of the RFP algorithm, it is necessary to cut the spectrum in several frequency ranges with only a few modes. This procedure makes the controller design more complicated. In the case of the DMPE algorithm, a single frequency range is sufficient to identify all the modes and the modal model identification 
can be largely automated. It also shows that the controller performances are closely linked to the modal amplitude values. In the case of modal amplitudes with a large real part, the controller performances are close to those numerically obtained. In the case of modal amplitudes with a large imaginary part, the real modal model doesn't take into account the phase shift and this error has a negative impact on the controller performances. Future works will deal with the control of the phase shift to ensure the performances of the controller even in the case of modal amplitude with higher imaginary part.

\section{References}

[1] A. Preumont. Vibration Control of Active Structures: An Introduction, Third Edition. In Vibration control of active structures: an introduction, third edition, volume 179 of Solid Mechanics and its Applications, pages 1-427. 2011.

[2] S. Prakash, T. G. Renjith Kumar, S. Raja, D. Dwarakanathan, H. Subramani, and C. Karthikeyan. Active vibration control of a full scale aircraft wing using a reconfigurable controller. Journal of Sound and Vibration, 361:32-49, 2016.

[3] Q. Zhang, C. Li, J. Zhang, and J. Jin. Active Vibration Control and Coupled Vibration Analysis of a Parallel Manipulator with Multiple Flexible Links. Shock and Vibration, page 7474085, 2016.

[4] A. Abu Hanieh and A. Preumont. Multi-axis vibration isolation using different active techniques of frequency reduction. Journal of Vibration and Control, 17(5):759-768, 2011.

[5] H. Yue, Y. Lu, Z. Deng, and H. Tzou. Experiments on vibration control of a piezoelectric laminated paraboloidal shell. Mechanical Systems And Signal Processing, 82:279-295, 2017.

[6] B. Chomette, D. Remond, S. Chesne, and L. Gaudiller. Semi-adaptive modal control of on-board electronic boards using an identification method. Smart Materials and Structures, 17(6), 2008.

[7] B. Chomette, S. Chesne, D. Remond, and L. Gaudiller. Damage reduction of on-board structures using piezoelectric components and active modal control-Application to a printed circuit board. Mechanical Systems and Signal Processing, 24(2):352-364, 2010.

[8] T. Kaizuka, N. Tanaka, and K. Nakano. Active control of sound transmission using structural modal filters. Journal of Sound and Vibration, 381:14-29, 2016.

[9] S. Benacchio, B. Chomette, A. Mamou-Mani, and F. Ollivier. Modal proportional and derivative state active control applied to a simplified string instrument. Journal of Vibration and Control, 22(18):3877-3888, 2016.

[10] S. Benacchio, A. Mamou-Mani, B. Chomette, and V. Finel. Active control and sound synthesis-two different ways to investigate the influence of the modal parameters of a guitar on its sound. Journal of The Acoustical Society of America, 139(3):1411-1419, 2016.

[11] S. Braghin, F. Cinquemani, and F. Resta. A new approach to the synthesis of modal control laws in active structural vibration control. Journal of Vibration and Control, 19(2):163-182, 2013.

[12] D. Formenti and M. Richardson. Parameter estimation from frequency response measurements using rational fraction polynomials (twenty years of progress). In Proceedings of IMAC-XX: Structural Dynamics Vols I And II, volume 4753, pages 373-382, 2002.

[13] G. Forsythe. Generation And Use Of Orthogonal Polynomials For Data-Fitting With A Digital Computer. Journal of the Society for Industrial and Applied Mathematics, 5(2):74-88, 1957.

[14] H. Van Der Auweraer and J. Leuridan. Multiple Input Orthogonal Polynomial ParameterEstimation. Mechanical Systems And Signal Processing, 1(3):259-272, 1987.

[15] S. Chesne, C. Jean-Mistral, and L. Gaudiller. Experimental identification of smart material coupling effects in composite structures. Smart Materials and Structures, 22(7):1-10, 2013.

[16] M. Porfiri, C. Maurini, and J. Pouget. Identification of electromechanical modal parameters of linear piezoelectric structures. Smart Material and Structures, 16(2):323-331, 2007. 
[17] C. Maurini, M. Porfiri, and J. Pouget. On the identification of modal couplings and inherent capacitances of piezoelectric structures - art. no. 65230U. In Lindner, DK, editor, Modeling, Signal Processing, and Control for Smart Structures 2007, volume 6523, page U5230, 2007.

[18] HH. Chen and F. Xu. A dual fitting algorithm for modal parameters identification. Mechanical Systems and Signal Processing, 17(3):713-721, 2003.

[19] M. El-Kafafy and P. Guillaume. Direct calculation of modal parameters from matrix orthogonal polynomials. Mechanical Systems and Signal Processing, 25(7):2375-2387, 2011.

[20] R.H. Levine. Modal parameter estimation for stable filters, 1996. US Patent 5579243.

[21] P. Mohanty, P. Reynolds, and A. Pavic. Automated Interpretation of Stability Plots for Analysis of a Non-Stationary Structure. In Proceedings of IMAC 25, Orlando, FL, USA, 2007.

[22] J. Lau, J. Lanslots, B. Peeters, and H. Van der Auweraer. Automatic modal analysis: Reality or myth? In Proceedings of IMAC 25, Orlando, FL, USA, 2007.

[23] M. Valasek and N. Olgac. Efficient Egenvalue Assignement for General Linear MIMO System. Automatica, 31(11):1605-1617, 1995.

[24] David G. Luenberger. Observing the state of a linear system. IEEE Transaction on Military Electronics, 8:74-80, 1964.

[25] R. Piéchaud. A Lightweight $\mathrm{C}++$ Framework for Real Time Active Control. In 16th Real Time Linux Workshop, Dusseldorf, Oct. 12 and 13, 2014. 